12,05

\title{
Природа открытия и модификации дираковской щели в аксионном антиферромагнитном топологическом изоляторе $\mathrm{MnBi}_{2} \mathrm{Te}_{4}$
}

\author{
(C) А.М. Шикин \\ Санкт-Петербургский государственный университет, \\ Санкт-Петербург, Россия \\ E-mail: ashikin@inbox.ru
}

Поступила в Редакцию 1 апреля 2020 г.

В окончательной редакции 1 апреля 2020 г.

Принята к публикации 9 апреля 2020 г.

Методом фотоэлектронной спектроскопии с угловым разрешением при фотовозбуждении лазерным излучением $(h v=6.3 \mathrm{eV})$ изучена модификация щели, открываемой в точке Дирака в аксионном антиферромагнитном топологическом изоляторе $\mathrm{MnBi}_{2} \mathrm{Te}_{4}$ при температурах выше и ниже температуры Нееля $(24.5 \mathrm{~K})$. Показано, что открываемая щель может иметь как аномально большую $(62-67 \mathrm{meV})$, так и значительно уменьшенную $(15-18 \mathrm{meV})$ величину на различных образцах $\mathrm{MnBi}_{2} \mathrm{Te}_{4}$ или различных участках одного и того же образца. В обоих случаях щель в точке Дирака остается открытой выше температуры Нееля. В результате проведенных исследований было выдвинуто предположение, что различие в величине щели, открываемой в точке Дирака, связано с проявлением эффекта фракционирования аксионного члена $\theta$. Величина щели $(62-67 \mathrm{meV})$, коррелирующая с результатами теоретических расчетов, соответствует состоянию с аксионным членом $\theta=\pi$. При этом экспериментальное наблюдение щели в точке Дирака $15-18 \mathrm{meV}$ соответствует эффективной модуляции аксионного члена $\theta=\pi / 4$ за счет генерации многоэлектронных хиральных спиновых флуктуаций и их взаимодействия при лазерном фотовозбуждении.

Ключевые слова: антиферромагнитный топологический изолятор, фотоэлектронная спектроскопия с угловым и спиновым разрешением, магнитная щель в точке Дирака.

DOI: 10.21883/FTT.2020.08.49617.068

\section{1. Введение}

Взаимосвязь между топологическими свойствами твердого тела и магнетизмом, характерная для магнитно-допированных топологических изоляторов (ТИ), играет существенную роль в формировании таких уникальных топологических квантовых эффектов, как квантовый аномальный эффект Холла [1-4] и топологический квантовый магнитоэлектрический эффект $[1,5,6]$, которые являются важными и высоко перспективными как для фундаментальной науки, так и для будущих технологических приложений, таких как топологическая бездиссипативная квантовая электроника и топологические квантовые вычисления. Эти эффекты сопровождаются открытием энергетической щели в точке Дирака, (возникающей в результате нарушения симметрии обращения времени вследствие формируемого магнитного упорядочения), внутри которой формируются краевые состояния, которые в свою очередь, и являются проводником краевых квантованных спиновых токов, лежащих в основе выше указанных эффектов.

При этом магнитные ТИ (см., например, [7-17]) можно одновременно рассматривать в качестве многообещающей платформы для реализации и непосредственного исследования таких фундаментальных квантовых эффектов, как формирование магнитного монополя и аксиона, фракционализации эффективного электрического заряда и квантования магнитного потока [1,18-26]. Эти эффекты влияют также на величину магнитной щели, открываемой в точке Дирака в магнитном ТИ, что может быть использовано, в свою очередь, в качестве индикатора данных уникальных топологических квантовых эффектов.

Некоторое время назад был успешно синтезирован магнитно-упорядоченный антиферромагнитный (АФМ) топологический изолятор со стехиометрией $\mathrm{MnBi}_{2} \mathrm{Te}_{4}$, который характеризуется уникальным сочетанием антиферромагнетизма и нетривиальных топологических свойств и который демонстрирует аномально большую энергетическую щель в точке Дирака (вплоть до $88 \mathrm{meV}$ [7]), что позволяет анализировать ее возможную модуляцию при реализации данных эффектов. Непосредственно после первоначального синтеза $\mathrm{MnBi}_{2} \mathrm{Te}_{4}$ были исследованы особенности электронной структуры данного уникального АФМ ТИ (как теоретически, так и экспериментально) и проанализированы его магнитные свойства [7-12]. Данное соединение можно рассматривать как образованное внедрением бислоя МnTe внутрь элементарной ячейки ТИ $\mathrm{Bi}_{2} \mathrm{Te}_{3}$, что приводит к формированию магнитно-упорядоченного слоя атомов $\mathrm{Mn}$ внутри каждого семислойника $\mathrm{MnBi}_{2} \mathrm{Te}_{4}$ с ферромагнитной $(Ф \mathrm{M})$ связью, ориентированной вдоль оси $(c)[7,8]$. При этом между соседними Мn-слоями формируется АФМ связь. То есть соседние $\mathrm{Mn}$ слои связаны антиферромагнитно вдоль направления перпендикулярно поверхности (111). В результате все магнитные момен- 
ты Mn-атомов упорядочены и выровнены относительно оси $(c)$, т.е. перпендикулярно поверхности (111). Нетривиальные поверхностные топологические состояния формируется для ТИ $\mathrm{MnBi}_{2} \mathrm{Te}_{4}$ инвертированием состояний $\mathrm{Bi} p p_{z}$ и Те $p_{z}$ в точке Г за счет сильной спинорбитальной связи, аналогично как и для $\mathrm{Bi}_{2} \mathrm{Te}_{3}$ [7-10]. Теоретические расчеты предсказывают для АФМ ТИ $\mathrm{MnBi}_{2} \mathrm{Te}_{4}$ аномально большую энергетическую щель в точке Дирака (до $88 \mathrm{meV}$ [7]), имеющую магнитный характер за счет обрыва АФМ-связи на поверхности при терминации поверхности ФМ слоем атомов Мn. В то же время, по результатам фотоэмиссионных исследований с угловым разрешением [7-11] экспериментально наблюдаемая щель для $\mathrm{MnBi}_{2} \mathrm{Te}_{4}$ может варьироваться от 50 до $100 \mathrm{meV}$, в зависимости от энергии фотонов и условий измерения. Кроме того, был выявлен неожиданный результат: несмотря на магнитную природу щели, открываемой в точке Дирака, щель остается открытой выше температуры магнитного упорядочения (температуры Нееля, которая для $\mathrm{MnBi}_{2} \mathrm{Te}_{4}$ соответствует $24.5 \mathrm{~K}[7,12])$. Интересно, что сохранение щели в точке Дирака выше температуры Нееля наблюдается также и для магнитно-допированных ТИ, в первую очередь для АФМ ТИ, допированных атомами $\mathrm{Gd}[16,17,27]$. Для ФМ ТИ, допированных атомами $\mathrm{Mn}$ и $\mathrm{V}$, также наблюдалось сохранение щели выше температуры Кюри [6,28-32]. При этом было высказано предположение [32-34], что для Мn-допированных ТИ формирование щели в точке Дирака связано с немагнитными эффектами гибридизации с $\mathrm{Mn} d$ состояний, которые пересекают топологические состояния в области точки Дирака. В противовес этому, для $\mathrm{MnBi}_{2} \mathrm{Te}_{4}$ (как было показано методами резонансной фотоэлектронной спектроскопии [7,35] и теоретическими исследованиями $[7,8])$ уровни $\mathrm{Mn} d$ расположены энергетически далеко от точки Дирака, что не может приводить к открытию немагнитной гибридизационной щели в точке Дирака. То есть вопрос о природе сохранения щели выше температуры Нееля в АФМ ТИ $\mathrm{MnBi}_{2} \mathrm{Te}_{4}$ остается открытым, и данная работа также будет посвящена, в том числе, и попыткам анализа причин сохранения щели выше температуры Нееля для данного АФМ ТИ.

Одновременно с этим, в последнее время в литературе появилась серия альтернативных работ [9,13-15], в которых для $\mathrm{MnBi}_{2} \mathrm{Te}_{4}$ были представлены результаты экспериментального наблюдения методом фотоэлектронной спектроскопии с угловым разрешением „бесщелевой“ дисперсии топологических состояний в области точки Дирака. Хотя в работах $[9,13]$ отмечалось, что и для этих экспериментов в измеряемых дисперсиях топологических состояний также можно выделить небольшую энергетическую щель в точке Дирака $(12-13.5 \mathrm{meV})$, которая также остается открытой выше температуры Нееля $[9,13]$. То есть в литературе существуют альтернативные измерения, показывающие либо аномально большую энергетическую щель в точке Дирака $(60-80 \mathrm{meV})$ [7-11] в соответствии с теоре- тическими расчетами, либо близкую к „безщелевой“ дисперсию топологических состояний [9,13-15] с существенно меньшей энергетической щелью в точке Дирака $(12-15 \mathrm{meV})[9,13]$.

При этом, согласно теоретическому моделированию, выполненному в работе [13], „бесщелевые“ дисперсии топологических состояний могут возникать в $\mathrm{MnBi}_{2} \mathrm{Te}_{4}$ из-за поверхностной магнитной реконструкции, которая приводит к существенному уменьшению эффективного магнитного момента перпендикулярного поверхности, в поверхностном Mn-слое, открывающем щель в точке Дирака. Это может быть связано: (a) с формированием латерального АФМ-взаимодействия в поверхностном магнитном слое (вместо латерального ФМ-упорядочения); $(b)$ - с поворотом магнитных моментов в поверхностном магнитном слое вдоль поверхности с соответствующим уменьшением эффективного момента перпендикулярно поверхности и $(c)-$ с формированием на поверхности магнитно- неупорядоченного парамагнитного слоя, т. е. „мертвого“ магнитного слоя. При этом возможности наблюдения аномально большой щели в точке Дирака в рамках данных моделей не предполагается. С другой стороны, магнитные измерения [7,35] свидетельствуют скорее о сохранении ФМ-упорядочения в верхнем магнитном слое и общем объемном АФМ-упорядочении перпендикулярно поверхности, в том числе и для образцов с малой щелью в точке Дирака, т.е. требуются другие альтернативные идеи, объясняющие одновременно существование как большой, так и малой щели, открываемой для АФМ ТИ $\mathrm{MnBi}_{2} \mathrm{Te}_{4}$.

В настоящей работе методами фотоэлектронной спектроскопии с угловым разрешением представлен детальный анализ открытия щели Дирака в стехиометрическом АФМ ТИ $\mathrm{MnBi}_{2} \mathrm{Te}_{4}$, выше и ниже температуры Нееля $(24.5 \mathrm{~K})$ и показана возможность экспериментального наблюдения (в одних и тех же экспериментальных условиях) как аномально большой $(62-67 \mathrm{meV})$, так и сушественно уменьшенной $(15-18 \mathrm{meV})$ энергетической щели в точке Дирака, как для различных образцов $\mathrm{MnBi}_{2} \mathrm{Te}_{4}$, так и различных участков поверхности одного и того же образца, неоднородного по поверхности. С другой стороны, в рамках работы предложены альтернативная идея открытия и модуляции величины щели в точке Дирака при различных температурах в рамках модели фракционализации аксионного члена для $\mathrm{MnBi}_{2} \mathrm{Te}_{4}$ при фотовозбуждении лазерным излучением и соответствующей фракционализации кванта магнитного потока, проявляющихся в экспериментальной наблюдаемой модуляции величины щели, открываемой в точке Дирака.

\section{2. Результаты}

Рис. $1, a, b$ (верхняя линия вставок) показывает сравнение дисперсионных зависимостей, измеренных методом 

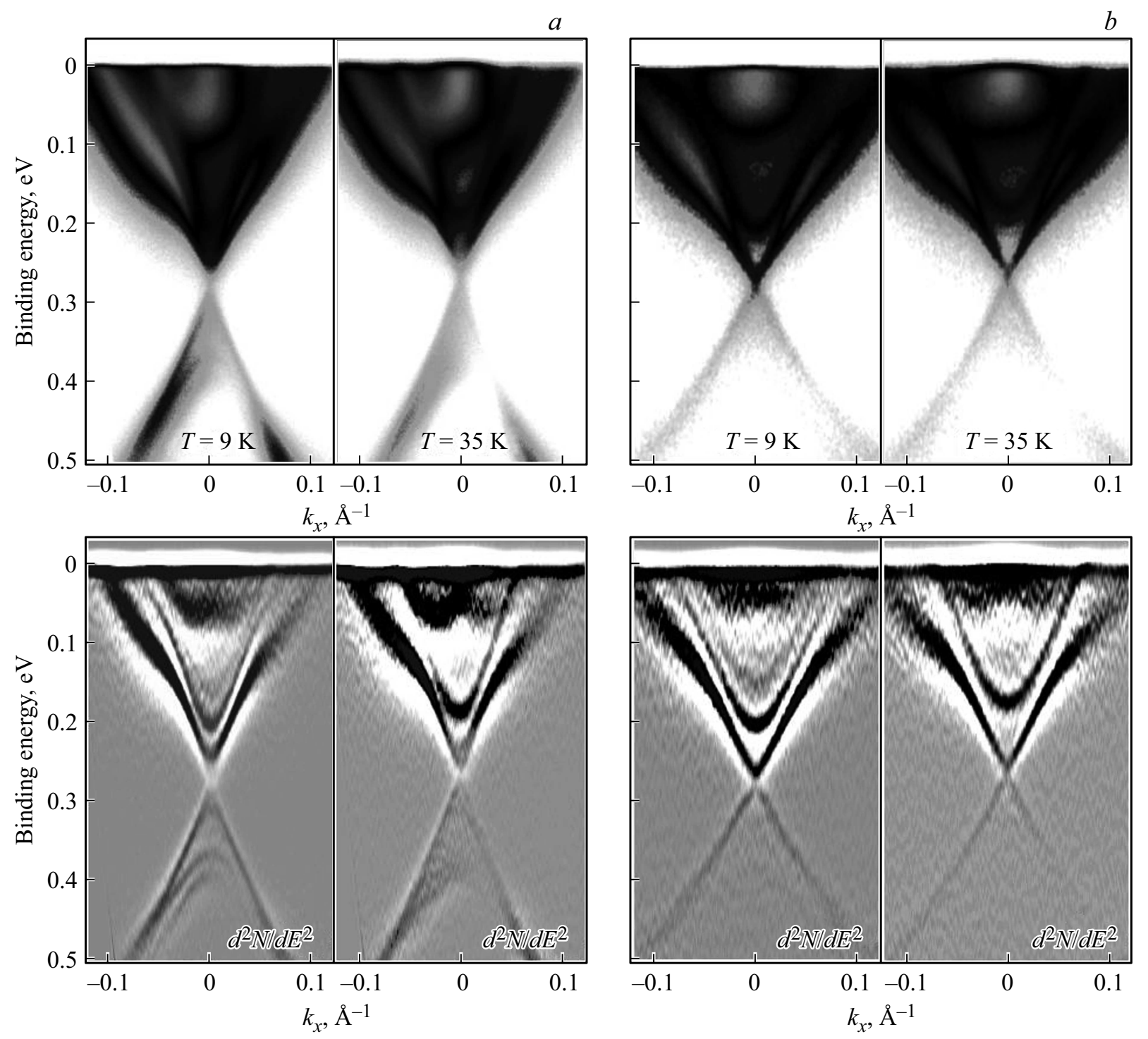

Рис. 1. $(a, b)$, верхние панели - дисперсии электронных состояний, измеренные для $\mathrm{MnBi}_{2} \mathrm{Te}_{4}$ при фотовозбуждении лазерным излучением (с энергией фотонов $6.3 \mathrm{eV})$ при температурах ниже $(9 \mathrm{~K})$ и выше температуры Нееля $(35 \mathrm{~K})$ для образцов с большой и малой щелью, открываемой в точке Дирака. $(a, b)$, нижние панели - то же самое, но в представлении $d^{2} N / d E^{2}$.

фотоэлектронной спектроскопии с угловым разрешением для $\mathrm{MnBi}_{2} \mathrm{Te}_{4}$ ниже и выше температуры Нееля $(24.5 \mathrm{~K}[7,12])$ с использованием $p$-поляризованного лазерного излучения с энергией фотонов $6.3 \mathrm{eV}$. Во втором ряду вставок показаны данные дисперсионные зависимости, представленные в форме второй производной $\left(d^{2} N / d E^{2}\right)$, для лучшей визуализации щели, открываемой в точке Дирака, и особенностей электронной структуры состояний валентной зоны и зоны проводимости. Дисперсионные зависимости, представленные на панелях $(a)$ и $(b)$, показывают положения краев состояний зоны проводимости и валентной зоны, которые расположены при температуре $9 \mathrm{~K}$ при энергиях связи около 0.22 и $0.36 \mathrm{~V}$ соответственно. При измерениях при температуре $35 \mathrm{~K}$ края зоны проводимости и валентной зоны расположены при энергиях связи приблизительно
0.19 и 0.38 С. Данное различие связано с обменным расщеплением состояний на краях зоны проводимости и валентной зоны ниже температуры Нееля (при 9K), за которым следует и соответствующее уменьшение фундаментально запрещенной зоны (более подробную информацию об этих состояниях и их изменениях с температурой см. в работе [36]). Топологические поверхностные дираковские состояния расположены внутри фундаментальной запрещенной зоны. Дисперсионные зависимости, представленные на панели $(a)$, характеризуются открытием энергетической щели в точке Дирака как ниже, так и выше температуры Нееля. Положение точки Дирака смещается в сторону уровня Ферми при более высоких температурах.

На рис. 2, $a$ представлены соответствующие энергетические распределения плотности топологических по- 


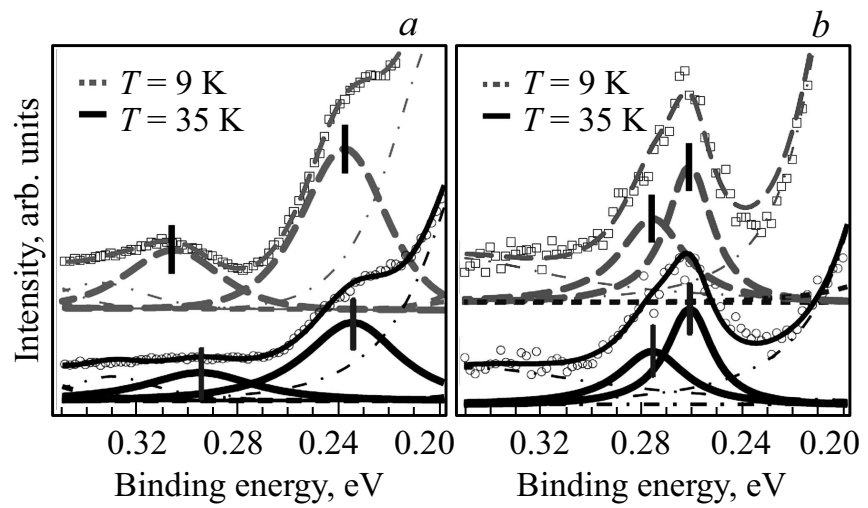

Pис. 2. $(a, b)$ - профили фотоэлектронных спектров, измеренные в области точки Дирака (в точке $\Gamma$ при $k_{\mathrm{II}}=0$ ) при температурах 9 и $35 \mathrm{~K}$ (серые и черные линии соответственно), с представлением разложения на спектральные компоненты, показывающие открытие большой и малой щели в точке Дирака и их изменение при температурах выше и ниже температуры Нееля.

верхностных состояний, измеренные непосредственно в области точки Дирака (в точке $\Gamma$ при $\left.k_{\mathrm{II}}=0\right)$ при температурах 9 и $35 \mathrm{~K}$ соответственно (см. также [35,36]). Хорошо видно значительное падение плотности состояний (фотоэмиссионного сигнала) при температуре $9 \mathrm{~K}$ в точке Дирака между максимумами (отмечены черными вертикальными линиями), свидетельствующее об открытии энергетической щели в точке Дирака. При этом видно, что щель остается открытой и при температурах выше температуры Нееля. Соответствующие оценки позволяют оценить величину открываемой щели при температуре $9 \mathrm{~K}$ на уровне около $67 \mathrm{meV}$ (при использовании р-поляризованного излучения). При температуре $35 \mathrm{~K}$ измеряемая величина щели немного уменьшается до $59 \mathrm{meV}$. Принимая во внимание ширину измеренных спектральных линий, неопределенность в измеренном значении щели в точке Дирака можно оценить на уровне $\pm 5 \mathrm{meV}$.

В то же время, образцы другого типа (см. рис. $1, b$ и $2, b)$, измеренные при тех же экспериментальных условиях при возбуждении лазерным излучением, демонстрируют принципиально отличающиеся дисперсионные зависимости (подобные так называемым „бесщелевым“ дисперсиям). На рис. 2, $b$ представлены соответствующие профили плотностей электронных состояний, измеренные в точке Дирака (при $\left.k_{\mathrm{II}}=0\right)$ для данного типа образцов, также при температурах ниже и выше температуры Нееля (см. также [35]). Данные энергетические профили имеют двухпиковую структуру, что позволяет выделить расщепление дираковских топологических состояний в точке Дирака, соответствующее открытию малой, но конечной величины щели в точке Дирака. Разложение на спектральные компоненты, которые также показаны на рис. $2, b$, позволяют оценить величину щели в точке Дирака на уровне $18-20 \mathrm{meV}$ с погрешностью не менее $\pm 7 \mathrm{meV}$. Причем формируемая щель в точке Дирака остается открытой и выше температуры Нееля, практически не изменяя своей величины. Данная оценка коррелирует со значением величины щели, оцененной в работе [9].

Интересно, что значение обменного расщепления для состояний зоны проводимости в области энергий связи $0.15-0.2 \mathrm{eV}$ (см. рис. 1, $a$ и $b$ ) имеет практически одинаковое значение для обоих типов образцов, характеризующихся диаметрально противоположными размерами щелей в точке Дирака (см. для сравнения [36]). Причем для обоих типов образцов данное расщепление схлопывается при температурах выше температуры Нееля. Это указывает на сходство магнитного АФМ-упорядочения для обоих типов образцов, независимо от величины щели в точке Дирака.

Для того чтобы подтвердить магнитную природу открытия щели в точке Дирака были проведены исследования спиновой поляризации перпендикулярно поверхности для состояний верхнего и нижнего дираковского конуса методом фотоэлектронной спектроскопии с угловым и спиновым разрешением. На рис. $3, a, b$ (верхние панели) показаны фотоэлектронные спектры со спиновым разрешением для направления спина перпендикулярно поверхности, измеренные в точке Дирака (при $k_{\text {II }}=0$ ) при температурах ниже и выше температуры Нееля (20 и 40 K) (см. также [35]). Компоненты с противоположным направлением спина по-

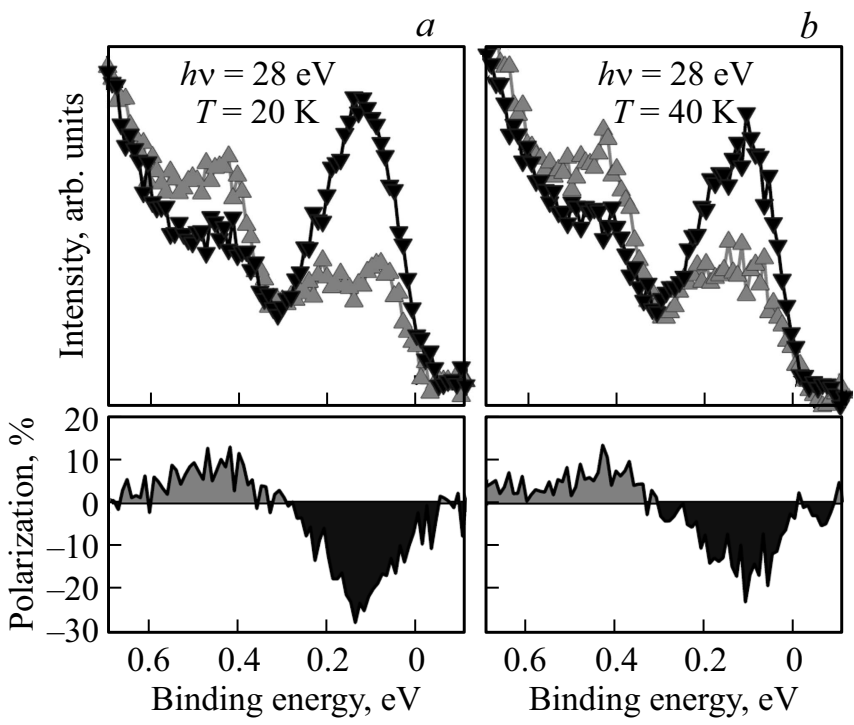

Рис. 3. Фотоэлектронные спектры со спиновым разрешением, измеренные в точке Дирака (при $\left.k_{\mathrm{II}}=0\right)$ с использованием $р$-поляризованного синхротронного излучения с энергией фотонов $28 \mathrm{eV}$ при температурах ниже $(T=20 \mathrm{~K})$ и выше $(T=40 \mathrm{~K})$ температуры Нееля. На нижних панелях представлена результирующая инверсная спиновая поляризация состояний верхнего и нижнего конуса Дираковских состояний (в совокупности со спиновой поляризацией состояний зоны проводимости и валентной зоны) относительно точки Дирака $\left(E_{B}=0.28 \mathrm{eV}\right)$. 
казаны разнонаправленными красными и синими треугольниками. Спектры измерены при фотовозбуждении $p$-поляризанным синхротронным излучением с энергией фотонов $28 \mathrm{eV}$.

На нижних панелях представлена соответствующая результирующая спиновая поляризация в направлении перпендикулярно поверхности для состояний верхнего и нижнего конуса дираковских состояний (в совокупности со спиновой поляризацией состояний зоны проводимости и валентной зоны) относительно точки Дирака $\left(E_{B}=0.28 \mathrm{eV}\right)$, измеренная ниже и выше температуры Нееля. Представленные спектры показывают инверсную спиновую поляризацию для состояний верхнего и нижнего конуса, что является характерным для щели, открываемой в точке Дирака магнитным взаимодействием с направлением магнитного момента перпендикулярно поверхности. Причем подобная инверсия спиновых состояний наблюдается как ниже, так и выше температуры Нееля. Аналогичная инверсия спиновой поляризация перпендикулярно поверхности наблюдается и для состояний зоны проводимости и валентной зоны, что говорит о включении в формируемое магнитное взаимодействие состояний зоны проводимости и валентной зоны.

Тот факт, что спиновая поляризация с направлением спинового момента перпендикулярно поверхности наблюдается также выше температуры Нееля (т.е. температуры дальнодействующего магнитного взаимодействия), может свидетельствовать о сохранении в некотором виде короткодействующего магнитного взаимодействия выше температуры Нееля. К сожалению энергетическое и угловое разрешение в данном эксперименте не позволяет разделять области поверхности или образцы с большой и малой величиной щели в точке Дирака. Тем не менее, интегрировано по поверхности ФМ-характер поверхностного магнитного упорядочения со спиновой ориентацией перпендикулярно поверхности наблюдается для всех измеренных образцов.

\section{3. Обсуждение результатов}

С одной стороны, чтобы объяснить тот экспериментальный факт, что щель в точке Дирака остается открытой выше температуры Нееля с сохранением спиновой инверсии дираковских состояний в верхнем и нижнем конусе (что характерно для щели магнитной природы), мы хотели бы предложить следующую идею. Если принять во внимание одновременно большое спинорбитальное взаимодействие в ТИ $\mathrm{MnBi}_{2} \mathrm{Te}_{4}$ и вертикальную анизотропию магнитных моментов, характерную для магнитного ТИ, то можно предположить, что взаимодействие между топологическими состояниями и магнитными моментами Мn-атомов должно иметь тенденцию к генерации спиновых флуктуаций с хиральной спиновой текстурой. То есть окружающая среда (топологический изолятор с большим спин-орбитальным взаимодействием и преимущественной спиновой ори- ентацией топологических состояний перпендикулярно импульсу в плоскости поверхности) стремится экранировать возбужденный магнитный момент, например, на магнитном дефекте или магнитном атоме, создавая при этом хиральную спиновую текстуру (посредством спиновых волн или спиновых флуктуаций). Формируемая текстура подобна магнитной текстуре мерона (блоховского типа) [37-39] с центральным магнитным моментом перпендикулярно плоскости (out-of-plane) и с постепенным поворотом магнитного момента в направлении поверхности (in-plane) с одновременным закручиванием вокруг центральной оси. В некоторой степени данная магнитная (спиновая) хиральная структура подобна той, которая была предложена в работе [40] для магнитных примесей внутри ТИ и является характерной для вихревых скирмионноподобных возбуждений (см. например, [37-39]). Возможность генерации скирминов непосредственно в $\mathrm{MnBi}_{2} \mathrm{Te}_{4}$ была экспериментально показана в работе [41].

Формирование подобных спиновых текстур в АФМ ТИ $\mathrm{Bi}_{2} \mathrm{Te}_{3}$, допированном атомами $\mathrm{Gd}$, было предложено в работе [27] для объяснения сохранения щели в точке Дирака выше температуры Нееля. Именно магнитная компонента перпендикулярно поверхности, формируемая в хиральных спиновых текстурах, и обуславливает открытие щели в точке Дирака магнитного характера. Спиновая ориентация в данной текстуре стабилизируется взаимодействием с топологическими поверхностными состояниями на периферии спиновой текстуры, и поэтому они могут формироваться выше температуры Нееля, открывая соответствующую щель в точке Дирака. Мы рассматриваем образование таких хиральных скирмионоподобных магнитных (спиновых) возбуждений как особый вид спиновых флуктуаций, возникающих в $\mathrm{MnBi}_{2} \mathrm{Te}_{4}$ выше температуры Нееля. Данные спиновые возбуждения могут генерироваться, по меньшей мере, вокруг магнитных межузельных дефектов, как показано в [41], или внутри слоев $\mathrm{Mn}-\mathrm{Te}$ в поверхностном слое, как это имеет место в [43]. Возможность возбуждения скирмионов в $\mathrm{MnBi}_{2} \mathrm{Te}_{4}$ и ТИ, легированном атомами Mn, была экспериментально подтверждена в работах $[41,42]$. Можно предположить, что лазерное возбуждение может дополнительно стимулировать генерацию таких хиральных скирмионоподобных возбуждений, как, например, в [44]. Эти возбуждения (или спиновые флуктуации) играют роль возникающего локального пространственно-модулированного магнитного поля, которое способно открыть щель в точке Дирака выше температуры Нееля.

С другой стороны, в данной статье мы попытаемся связать различные величины щелей в точке Дирака, наблюдаемые в фотоэлектронных спектрах для АФТ ТИ $\mathrm{MnBi}_{2} \mathrm{Te}_{4}$ (рис. 1 и 2), с модуляцией аксионного члена (axion term $\theta$ ), характерного для данного соединения, при различных граничных условиях, проявляемых в эксперименте. Известно, что AFM ТИ при $\theta \neq 0$ характеризуются дробным квантовым эффектом Холла 
(half-quantized QHE), что связывается с формированием аксионного члена $\frac{\theta}{2 \pi} \frac{e^{2}}{h c}$, обуславливающего в заимосвязь электрического и магнитного полей при взаимодействии с АФМ ТИ. Индуцированная намагниченность и холловская проводимость в рамках данного эффекта являются квантуемыми и определяются выражением $\frac{\theta e^{2}}{2 \pi h c}(E B)$, где для топологического изолятора $\theta= \pm \pi$ (модуль $2 \pi$ ) и $\theta=0$ для тривиального изолятора. Аксионный член (иногда называют axion angle $\theta$ ) проявляет себя на границе между двумя изоляторами, характеризующимися различными объемными значениями $\theta$, где имеется высокий градиент поля $\theta(r)$. Например, как это имеет место на границе АФМ ТИ (с $\theta=\pi$ ) и вакуума (который является тривиальным изолятором с $\theta=0)$, см., например $[1-3,19,54]$. При нарушении симметрии обращения времени (например, в магнитном ТИ), аксионный член может принимать произвольное значение, в зависимости от конкретных граничных условий и магнитных свойств материала.

При этом фундаментальным свойством аксионной среды с $\theta \neq 0$ является эффект Виттена, т.е. возможность формирования состояния магнитного монополя (или диона) в аксионной среде, характеризующегося квантом магнитного потока $\Phi_{0}=\frac{h c}{e}($ при $\theta=\pi)$, который несет на себе также дробный электрический заряд $\pm \frac{e \theta}{2 \pi}[1,18-26,45,46,54]$. Данная идея была развита и успешно применена в гетероструктурах на основе сверхпроводника и ТИ (см., например, $[22,23,25,26])$ с использованием идеи формирования монополей (с дробным эффективным зарядом) на противоположных поверхностях сверхпроводника, контактирующего с ТИ, связанных посредством вихревой струны (магнитного вихря) с эффективным квантом магнитного потока $\frac{\Phi_{0}}{2}=\frac{\theta}{2 \pi} \frac{h c}{e}$. Аналогичный результат был получен для АФМ или ионных одномерных цепочек $[46,47]$ и далее распространен на АФМ ТИ [45,54], которые также характеризуются индуцированным зарядом (электрической поляризацией) на концах цепочки или противоположных поверхностях ТИ, определяемым как $\pm e\left(n+\frac{\theta}{2 \pi}\right)$, где $n$ целое, и соответствующим эффективным аксионным членом, пропорциональным $\pm \frac{\theta}{2 \pi}$ на противоположных поверхностях АФМ ТИ. Таким образом, аксионное поле в АФМ ТИ обусловливает индукцию на противоположных поверхностях ТИ электрическую поляризацию противоположного знака $\left(+\frac{e}{2}\right.$ and $\left.-\frac{e}{2}\right)[1,18,45,46]$, что и обуславливает реализацию дробного квантового эффекта Холла и дробного магнетоэлектрического эффекта, приводя к открытию щели в точке Дирака, модулируемой индуцированным эффективным магнитным полем на границах АФМ ТИ.

При этом формированию дионоподобной связи описываемой в рамках эффекта Виттена с дробным зарядом $\left( \pm \frac{e}{2}\right)$ должна соответствовать АФМ-цепочка с четным числом атомов [46] или 3D АФМ ТИ с четным числом слоев по толщине [45], т.е. с противоположно направленными магнитными моментами на концах цепочки или противоположных поверхностях АФМ ТИ $[45,46]$. То есть если магнитные моменты на противоположных поверхностях противоположно направлены, то формируемое взаимодействие описывается на языке формирования дробного аксионного члена $\frac{\theta}{2 \pi}$. Если же магнитные моменты на противоположных поверхностях практически не связаны, то дионо-подобная связь не возникает, и аксионный член определяется как $\frac{\theta}{\pi}$. В соответствии с этим изменяется и эффективный квант магнитного потока (magnetic quantum flux) $\Phi=\frac{\Phi_{0}}{2}=\frac{1}{2} \frac{h c}{e}$ или $\Phi=\Phi_{0}=\frac{h c}{e}$.

По аналогии с системами на основе сверхпроводников, в АФМ ТИ роль монополеподобных структур, связанных посредством вихревой струны, могут играть хиральные скирмионо- или мероноподобные магнитные возбуждения, генерируемые на противоположных поверхностях и характеризующиеся противоположными магнитными моментами и противоположной спиновой хиральностью. При этом связь между поверхностными возбуждениями с противоположными магнитными (спиновыми) моментами может осуществляться посредством спиновой волны, формируемой в последовательности АФМ-слоев вдоль оси $(c)$, которая и играет роль вихревой (спиновой) струны (или „червоточины“ как в [51]). Эффективную генерацию подобной спиновой волны может стимулировать фотовозбуждение лазерным излучением. При энергии фотонов $6.3 \mathrm{eV}$ кинетическая энергия электронов, возбуждаемых с уровня Ферми, соответствует $1.2 \mathrm{eV}$, а электронов вблизи точки Дирака - $0.9 \mathrm{eV}$. Это соответствует длине волны около $11-13 \AA\left(\lambda=\sqrt{150 / E_{\mathrm{kin}}(\mathrm{eV})}\right)$, что коррелирует с периодом необходимых осцилляций спиновой плотности в АФМ периодичности Мn-слоев в $\mathrm{MnBi}_{2} \mathrm{Te}_{4}(12 \AA)$.

Но при этом возможны и более сложные многочастичные взаимодействия между формируемыми поверхностными магнитными возбуждениями, приводящие к дальнейшей фракционализации аксионного члена. Например, если предположить некоторый сдвиг локализации поверхностных топологических состояний в сторону второго более низко лежащего магнитного слоя, который характеризуется противоположной ориентацией магнитного момента по отношению к верхнему слою, то это может приводить к взаимно компенсирующему влиянию магнитных моментов в данных слоях на топологические состояния. (Такая возможность была показана в работе [35] за счет частичного увеличения ван-дер-Ваальсового расстояния между слоями). При этом формируемое магнитное взаимодействие можно представить как взаимодействие магнитных флуктуаций (мероно-подобного типа) противоположной хиральности и магнитной направленности, приводящее к соответствующей модуляции аксионного члена.

Известно, что мерон (блоховского типа) - это магнитная текстура хирального типа, которая может быть представлена как половина скирмиона и которая характеризуется дробным топологическим числом 
или топологическим зарядом $\pm \frac{1}{2}$ инверсного знака для противоположной магнитной ориентации в центре текстуры [37-39,48]. Экспериментальное подтверждение существования меронов и мероновых пар (и соответствующих топологических спиновых текстур) в хиральных магнитных структурах представлено в работах $[38,49,50]$. При этом отмечается энергетическая выгодность формирования бимероновых пар $[38,49]$ с противоположными направлениями центрального магнитного момента и хиральности.

Можно ожидать, что взаимодействие двух мероноподобных магнитных возбуждений противоположной магнитной направленности и хиральности должно сопровождаться дополнительной фракционализацией аксионного члена $\theta=\pi / 4$, которое может проявлять себя в соответствующем уменьшении щели в точке Дирака. В результате таких бимеронных взаимодействий основное состояние становится двукратно вырожденным с характерным квантом магнитного потока, равным $\Phi_{0} / 4$. Это должно приводить к соответствующему четырехкратному уменьшению величины щели в точке Дирака (по сравнению со случаем, характеризуемым для ситуации с $\theta=\pi$ ). Возможность формирования аксионного члена типа $\theta=\pi / 4$ показана в работах $[23,25,52,53,55]$, предполагая взаимодействие подобного типа многочастичных возбуждений с топологическим числом (зарядом) $\pm 1 / 2$. При этом фермионоподобные квазичастицы (представителем которых является мерон) в соответствии с теорией Виттена несут на себе дробный заряд $q= \pm e / 2$. Поэтому взаимодействие двух фермионоподобных возбуждений с эффективным зарядом $q= \pm e / 2$ (т.е. $(+1 / 2) \uparrow$ and $(-1 / 2) \downarrow)$ должно приводить к формированию квазичастицы, влияние которой на аксионный член должно приводить к его модуляции типа $\theta=q^{2} \pi=\pi / 4$ [53] (по аналогии с формированием куперовской пары в сверхпроводнике). Формирование куперовских пар в $\left(p_{x}+i p_{y}\right)$-сверхпрововодниках также приводит к формированию аксионного члена $\theta=\pi / 4$ с соответствующим влиянием на величину и структуру сверхпроводящей щели [56]. Взаимодействия подобного типа возможны и при взаимодействиях типа „частица-дырка“ [53]. То есть формирование многочастичных возбуждений, описываемых на языке формирования дробного аксионного члена типа $\theta=\pi / n$, может происходить непосредственно под действием лазерного фотовозбуждения.

В соответствии с вышеизложенным, можно предположить, что формирование щели в точке Дирака с величиной 62-67 meV соответствует эффективному значению аксионного члена $\theta=\pi$. При этом формирование щели 15-18 meV должно соответствовать случаю генерации на поверхности АФМ ТИ $\mathrm{MnBi}_{2} \mathrm{Te}_{4}$ бимеронного возбуждения с фракционализацией аксионного члена $\theta=\pi / 4$ и соответствующему четырехкратному уменьшениию величины щели в точке Дирака.

Так как генерация подобных магнитных мероноподобных возбуждений и их взаимодействие не опреде- ляются дальнодействующим магнитным упорядочением, то щель в точке Дирака, формируемая подобными взаимодействиями, остается открытой выше температуры Нееля. При этом взаимосвязь мероноподобных возбуждений, как уже отмечалось, фактически осуществляется посредством соответствующих спиновых волн, генерируемых при лазерном фотовозбуждении, с длиной волны определяемой периодичностью АФМ-взаимодействия в $\mathrm{MnBi}_{2} \mathrm{Te}_{4}$, приводя к модуляции эффективного аксионного поля $\theta=\pi / 4$. С другой стороны, при возбуждении синхротронным излучением с энергией фотонов более 20-50 eV длина волны возбуждаемых фотоэмиссией электронов существенно уменьшается, что приводит к уменьшению эффективности бимеронных возбуждений. Поэтому вероятность наблюдения в фотоэмиссионных спектрах уменьшенной щели в точке Дирака (15-18 meV), соответствующей формированию аксионного члена $\theta=\pi / 4$, существенно уменьшается, что и наблюдается в экспериментах с использованием синхротронного излучения. При возбуждении синхротронным излучением наблюдается в основном щель более 60-70 meV [7,8,10,11]. В то время как при возбуждении лазерным излучением более часто регистрируется щель $12-18 \mathrm{meV}[9,13-15]$.

При этом следует отметить, что из вышеизложенного рассмотрения следует, что в АФТ ТИ должно иметь место также формирование дробного аксионного члена типа $\theta=\pi / 2$. Что должно сопровождаться открытием щели в точке Дирака промежуточной величины (около 30-35 meV). Для подтверждения данного предположения нужны дополнительные экспериментальные исследования.

\section{4. Заключение}

Методом фотоэлектронной спектроскопии с угловым разрешением при фотовозбуждении лазерным излучением $(h v=6.3 \mathrm{eV})$ была изучена модификация щели, открываемой в точке Дирака в аксионном АФМ-топологическом изоляторе $\mathrm{MnBi}_{2} \mathrm{Te}_{4}$ ниже $(13$ и $20 \mathrm{~K})$ и выше $(35$ и $40 \mathrm{~K})$ температуры Нееля. Нам удалось выделить в измеренных дисперсиях топологических поверхностных состояний энергетическую щель в точке Дирака как аномально большой $(62-67 \mathrm{meV})$, так и значительно уменьшенной (15-18 meV) величины, наблюдаемой на различных образцах $\mathrm{MnBi}_{2} \mathrm{Te}_{4}$ или различных участках одного и того же образца. В обоих случаях щель в точке Дирака оставалась открытой выше температуры Нееля.

Спектры со спиновым разрешением показывают инверсию спиновой поляризации в направлении перпендикулярно поверхности для верхнего и нижнего конуса дираковских топологических состояний, что характерно для магнитной природы щели, открываемой в точке Дирака. Причем данная спиновая поляризация Дираковского конуса топологических состояний остается неиз- 
менной выше температуры Нееля, что свидетельствует о сохранении магнитной природы (короткодействующего типа) и выше температуры дальнодействующего магнитного упорядочения. Это может осуществляться посредством генерации хиральных спиновых флуктуацияй (мероно-подобного типа), которые связывают дираковские состояния с противоположным импульсом и ориентацией спина с генерируемым локальным магнитным полем, что и открывает щель в точке Дирака

В результате проведенных исследований была выдвинута новая альтернативная идея открытия щели в точке Дирака и наблюдения обоих типов щелей в $\mathrm{MnBi}_{2} \mathrm{Te}_{4}$ при различных условиях на поверхности образца. Различие в величине щели, открываемой в точке Дирака (62-67 meV и 15-18 meV), связывается с проявлением эффекта фракционирования аксионного члена $\theta$, возникающего при лазерном возбуждении. При этом щель в точке Дирака, полученная из теоретических расчетов и экспериментальных исследований $(62-88 \mathrm{meV})$ для АФМ TI $\mathrm{MnBi}_{2} \mathrm{Te}_{4}$, соответствует состоянию с аксионным членом $\theta=\pi$. Экспериментальное наблюдение щели в точке Дирака величины $15-18 \mathrm{meV}$ coответствует эффективной модуляции аксионного члена $\theta=\pi / 4$, возникающей при лазерном возбуждении, за счет многоэлектронных эффектов хиральных магнитных возбуждений (флуктуаций).

Предполагается, что существенную роль при реализации эффектов фракционализации аксионного члена играет генерация спиновых волн с длиной волны, соответствующей АФМ периодичности в структуре АФМ ТИ $\mathrm{MnBi}_{2} \mathrm{Te}_{4}$, при фотовозбуждении электронов с уровня Ферми и области вблизи точки Дирака.

\section{Финансирование работы}

Работа выполнена в рамках финансовой поддержки по гранту на НИР Санкт-Петербургского государственного университета (Pure 1D 40990069) и гранта РНФ № 1812-00062.

\section{Конфликт интересов}

У автора нет конфликта интересов.

\section{Список литературы}

[1] X.-L. Qi, T.L. Hughes, S.-C. Zhang. Phys. Rev. B 78, 195424 (2008).

[2] R. Yu, W. Zhang, H.-J. Zhang, S.-C. Zhang, X. Dai, Z. Fang. Science 329, 61 (2010).

[3] C.-Z. Chang, J. Zhang, X. Feng, J. Shen, Z. Zhang, M. Guo, K. Li, Y. Ou, P. Wei, L.-L. Wang, Z.-Q. Ji, Y. Feng, S. Ji, X. Chen, J. Jia, X. Dai, Z. Fang, S.-C. Zhang, K. He, Y. Wang, L. Lu, X.-C. Ma, Q.-K. Xue. Science 340, 167 (2013).

[4] C.-Z. Chang, W. Zhao, D.Y. Kim, H. Zhang, B. A. Assaf, D. Heiman, S.-C. Zhang, C. Liu, M.H.W. Chan, J.S. Moodera. Nature Mater. 14, 473 (2015).
[5] J. Wang, B. Lian, X.-L. Qi, S.-C. Zhang. Phys. Rev. B 92, 081107 (2015).

[6] Y.L. Chen, J.-H. Chu, J.G. Analytis, Z.K. Liu, K. Igarashi, H.-H. Kuo, X.L. Qi, S.K. Mo, R.G. Moore, D.H. Lu, M. Hashimoto, T. Sasagawa, S.C. Zhang, I.R. Fisher, Z. Hussain, Z.X. Shen. Science 329, 659 (2010).

[7] M.M. Otrokov, I.I. Klimovskikh, H. Bentmann, D. Estyunin, A. Zeugner, Z.S. Aliev, S. Gaß, A.U.B. Wolter, A.V. Koroleva, A.M. Shikin, M. Blanco-Rey, M. Hoffmann, I.P. Rusinov, A.Y. Vyazovskaya, S.V. Eremeev, Y.M. Koroteev, V.M. Kuznetsov, F. Freyse, J. Sánchez-Barriga, I.R. Amiraslanov, M.B. Babanly, N.T. Mamedov, N.A. Abdullayev, V.N. Zverev, A. Alfonsov, V. Kataev, B. Büchner, E.F. Schwier, S. Kumar, A. Kimura, L. Petaccia, G. Di Santo, R.C. Vidal, S. Schatz, K. Kißner, M. Ünzelmann, C.H. Min, S. Moser, T.R.F. Peixoto, F. Reinert, A. Ernst, P.M. Echenique, A. Isaeva, E.V. Chulkov. Nature 576, 416 (2019).

[8] D. Zhang, M. Shi, T. Zhu, D. Xing, H. Zhang, J. Wang. Phys. Rev. Lett. 122, 206401 (2019).

[9] J. Li, Y. Li, S. Du, Z. Wang, B.-L. Gu, S.-C. Zhang, K. He, W. Duan, Y. Xu. Sci. Adv. 5, eaaw5685 (2019).

[10] Y. Gong, J. Guo, J. Li, K. Zhu, M. Liao, X. Liu, Q. Zhang, L. Gu, L. Tang, X. Feng, D. Zhang, W. Li, C. Song, L. Wang, P. Yu, X. Chen, Y. Wang, H. Yao, W. Duan, Y. Xu, S.C. Zhang, X. Ma, Q.-K. Xue, K. He, Chin. Phys. Lett. 36, 076801 (2019).

[11] S.H. Lee, Y. Zhu, Y. Wang, L. Miao, T. Pillsbury, H. Yi, S. Kempinger, J. Hu, C.A. Heikes, P. Quarterman, W. Ratcliff, J.A. Borchers, H. Zhang, X. Ke, D. Graf, N. Alem, C.Z. Chang, N. Samarth, Z. Mao. Phys. Rev. Res. 1, 012011 (2019).

[12] Z.S. Aliev, I.R. Amiraslanov, D.I. Nasonova, A.V. Shevelkov, N.A. Abdullayev, Z.A. Jahangirli, E.N. Orujlu, M.M. Otrokov, N.T. Mamedov, M.B. Babanly, E.V. Chulkov. J. Alloys Comp. 789, 443 (2019).

[13] Y.-J. Hao, P. Liu, Y. Feng, X.-M. Ma, E. F. Schwier, M. Arita, S. Kumar, C. Hu, R. Lu, M. Zeng, Y. Wang, Z. Hao, H.-Y. Sun, K. Zhang, J. Mei, N. Ni, L. Wu, K. Shimada, C. Chen, Q. Liu, C. Liu. Phys. Rev. X 9, 041038 (2019).

[14] Y.J. Chen, L.X. Xu, J.H. Li, Y.W. Li, H.Y. Wang, C.F. Zhang, H. Li, Y. Wu, A.J. Liang, C. Chen, S.W. Jung, C. Cacho, Y.H. Mao, S. Liu, M.X. Wang, Y.F. Guo, Y. Xu, Z.K. Liu, L.X. Yang, Y.L. Chen. Phys. Rev. X 9, 041040 (2019).

[15] P. Swatek, Y. Wu, L.-L. Wang, K. Lee, B. Schrunk, J. Yan, A. Kaminski. arXiv:1907.09596 (2019).

[16] A.M. Shikin, D.A. Estyunin, Y.I. Surnin, A.V. Koroleva, E.V. Shevchenko, K.A. Kokh, O.E. Tereshchenko, S. Kumar, E.F. Schwier, K. Shimada, T. Yoshikawa, Y. Saitoh, Y. Takeda, A. Kimura. Sci. Rep. 9, 4813 (2019).

[17] S.O. Filnov, Y.A. Surnin, A.V. Koroleva, I.I. Klimovskikh, D.A. Estyunin, A.Y. Varykhalov, K.A. Bokai, K.A. Kokh, O.E. Tereshchenko, V.A. Golyashov, E.V. Shevchenko, A.M. Shikin. J. Exp. Theor. Phys. 129, 404 (2019).

[18] X.-L. Qi, R. Li, J. Zang, S.-C. Zhang. Science 323, 1184 (2009).

[19] X.-L. Qi, S.-C. Zhang. Rev. Mod. Phys. 83, 1057 (2011).

[20] E. Witten. Phys.Lett. B 86, 283 (1979).

[21] F. Wilczek. PRL 48, 1144 (1982).

[22] G. Rosenberg, M. Franz. Phys. Rev. B 82, 035105 (2010).

[23] F.S. Nogueira, Z. Nussinov, J. van den Brink. Phys. Rev. Lett. 117, 167002 (2016).

[24] F. Wilczek. RL 49, 957 (1982). 
[25] F.S. Nogueira, Z. Nussinov, J. van den Brink. Phys. Rev. Lett. 121, 227001 (2018).

[26] F.S. Nogueira, Z. Nussinov, J. van den Brink. PRD 94, 085003 (2010).

[27] A.M. Shikin, D.A. Estyunin, A.V. Koroleva, D.A. Glazkova, T.P. Makarova, S.O. Filnov. Phys. Solid State 62, 338 (2020).

[28] S.-Y. Xu, M. Neupane, C. Liu, D. Zhang, A. Richardella, L. Andrew Wray, N. Alidoust, M. Leandersson, T. Balasubramanian, J. Sánchez-Barriga, O. Rader, G. Landolt, B. Slomski, J. Hugo Dil, J. Osterwalder, T.-R. Chang, H.-T. Jeng, H. Lin, A. Bansil, N. Samarth, M. Zahid Hasan. Nature Phys. 8, 616 (2012).

[29] A.M. Shikin, A.A. Rybkina, D.A. Estyunin, D.M. Sostina, I.I. Klimovskikh, V.Y. Voroshnin, A.G. Rybkin, K.A. Kokh, O.E. Tereshchenko, L. Petaccia, G. Di Santo, A. Kimura, P.N. Skirdkov, K.A. Zvezdin, A.K. Zvezdin. Sci. Rep. 8, 6544 (2018).

[30] A.M. Shikin, A.A. Rybkina, D.A. Estyunin, D.M. Sostina, V.Y. Voroshnin, I.I. Klimovskikh, A.G. Rybkin, Y.A. Surnin, K.A. Kokh, O.E. Tereshchenko, L. Petaccia, G. Di Santo, P.N. Skirdkov, K.A. Zvezdin, A.K. Zvezdin, A. Kimura, E.V. Chulkov, E.E. Krasovskii. Phys. Rev. B 97, 245407 (2018).

[31] A.M. Shikin, A.A. Rybkina, I.I. Klimovskikh, M.V. Filianina, K.A. Kokh, O.E. Tereshchenko, P.N. Skirdkov, K.A. Zvezdin, A.K. Zvezdin. Appl. Phys. Lett. 109, 222404 (2016).

[32] J. Sánchez-Barriga, A. Varykhalov, G. Springholz, H. Steiner, R. Kirchschlager, G. Bauer, O. Caha, E. Schierle, E. Weschke, A.A. Ünal, S. Valencia, M. Dunst, J. Braun, H. Ebert, J. Minár, E. Golias, L.V. Yashina, A. Ney, V. Holý, O. Rader. Nature Commun. 7, 10559 (2016).

[33] A.M. Black-Schaffer, A.V. Balatsky, J. Fransson. Phys. Rev. B 91, 201411 (2015).

[34] Y. Xu, J. Chiu, L. Miao, H. He, Z. Alpichshev, A. Kapitulnik, R.R. Biswas, L.A. Wray. Nature Commun. 8, 14081 (2017).

[35] A.M. Shikin, D.A. Estyunin, I.I. Klimovskikh, S.O. Filnov, E.F. Schwier, S. Kumar, K. Myamoto, T. Okuda, A. Kimura, K. Kuroda, K. Yaji, S. Shin, Y. Takeda, Y. Saitoh, Z.S. Aliev, N.T. Mamedov, I.R. Amiraslanov, M.B. Babanly, M.M. Otrokov, S.V. Eremeev, E.V. Chulkov. arXiv:2004.04802v1[cond-mat. mtrl-sci] (2020).

[36] D.A. Estyunin, I.I. Klimovskikh, A.M. Shikin, E.F. Schwier, M.M. Otrokov, A. Kimura, S. Kumar, S.O. Filnov, Z.S. Aliev, M.B. Babanly, E.V. Chulkov. APL Mater. 8, 021105 (2020).

[37] S.-Z. Lin, A. Saxena, C. D. Batista. Phys. Rev. B 91, 224407 (2015).

[38] N. Gao S.-G. Je, M.-Y. Im, J.W. Choi, M. Yang, Q. Li, T.Y. Wang, S. Lee, H.-S. Han, K.-S. Lee, W. Chao, C. Hwang, J. Li, Z.Q. Qiu. Nature Commun. 10, 5603 (2019).

[39] N. Nagaosa, Y. Tokura. Nature Nanotechnology 8, 899 (2013).

[40] Q. Liu, C.-X. Liu, C. Xu, X.-L. Qi, S.-C. Zhang. Phys. Rev. Lett. 102, 156603 (2009).

[41] J.Q. Yan, D. Pajerowski, L. Ke, A. M. Nedic, Y. Sizyuk, E. Gordon, P.P. Orth, D. Vaknin, R.J. Mc Queeney. arXiv:1908.02332 [cond-mat.str-el].

[42] C. Liu, Y. Zang, W. Ruan, Y. Gong, K. He, X. Ma, Q.-K. Xue, Y. Wang. Phys. Rev. Lett. 119, 176809 (2017).

[43] Q.L. He, G. Yin, A.J. Grutter, L. Pan, X. Che, G. Yu, D.A. Gilbert, S.M. Disseler, Y. Liu, P. Shafer, B. Zhang, Y. Wu, B.J. Kirby, E. Arenholz, R.K. Lake, X. Han, K.L. Wang. Nature Commun. 9, 2767 (2018).

[44] N. Ogawa, S. Seki, Y. Tokura. Sci. Rep. 5, 9552 (2015).
[45] N.P. Armitage, L. Wu. Sci.Post Phys. 6, 046 (2019).

[46] I. Affleck. PRL 57, 1048 (1986).

[47] V.P. Su, J.R. Schrieffer, A.J. Heeger. PRL 42, 1698 (1979); PRB22, 2099 (1980).

[48] S.Z. Liu, A. Saxena, C. D. Batista. PRB 91, 224407 (2015).

[49] A. Tan J. Li, A. Scholl, E. Arenholz, A.T. Young, Q. Li, C. Hwang, Z.Q. Qiu. PRB 94, 014433 (2016).

[50] X.Z. Yu, W. Koshibae, Y. Tokunaga, K. Shibata, Y. Taguchi, N. Nagaosa, Y. Tokura. Nature 564, 95 (2018).

[51] G. Rosenberg, H.-M. Guo, M. Franz. PRB 82, 041104(R) (2010).

[52] C. Weeks, G. Rosenberg, B. Seradjeh, M. Franz. Nature Phys. 3, 796 (2007).

[53] Y. Ran, A. Vishwanath, D-H. Lee. PRL 101, 086801 (2008).

[54] R.S.K. Mong, A.M. Essin, J.E. Moore. PRB 81, 245209 (2010).

[55] B. Swingle, M. Barkeshli, J. McGreevy, T. Senthil. Phys. Rev. B 83, 195139 (2011).

[56] N. Read, D. Green. PRB 61, 10267 (2000).

Редактор Т.Н. Василевскал 